\title{
Thoracic splenosis: Mimicry of a neurogenic tumor
}

\author{
Costas S. Bizekis, MD, Bradley Pua, BA, and Lawrence R. Glassman, MD, New York, NY
}

$\mathrm{T}$ horacic splenosis refers to a rare condition of ectopic splenic tissue in the left hemithorax. Patients with this condition are often asymptomatic, with a history of thoracoabdominal trauma to the spleen and left hemidiaphragm. Nodules are usually an incidental finding on chest radiography or computed tomography (CT). Most often, thoracic splenosis is diagnosed intraoperatively. When splenosis is suspected, nuclear scanning methods might aid in preoperative diagnoses, thus avoiding the resection of the benign mass and its operative risks. To date, only 26 cases of thoracic splenosis have been reported in the literature. ${ }^{1}$ The following is a case report of thoracic splenosis mimicking a neurogenic tumor.

\section{Clinical Summary}

The patient is a 47-year-old man, a recovering drug and alcohol addict who had a laparotomy and splenectomy in 1982 after a
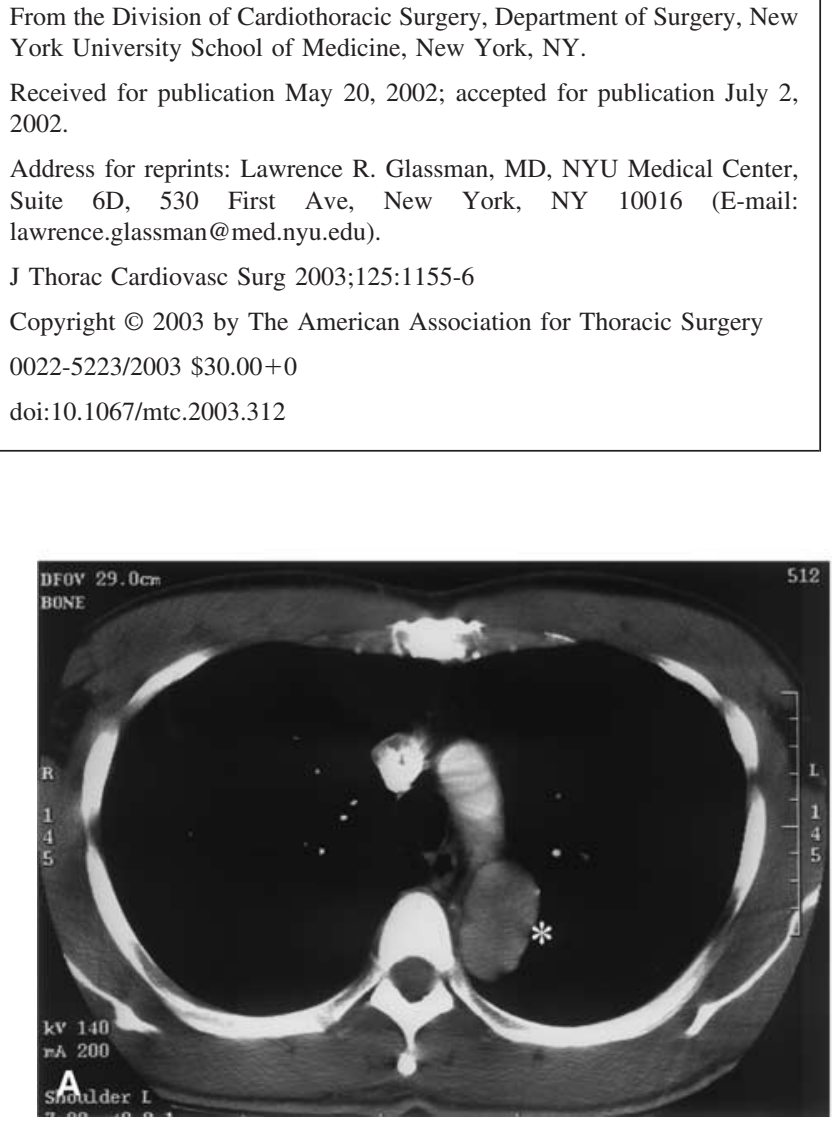

gunshot wound. During a routine admission, abnormalities were noted on a chest $\mathrm{x}$-ray film; this $\mathrm{x}$-ray film prompted a chest CT scan, and on this examination, 2 abnormal masses were noted (Figure 1). One mass was located in the left paraspinal region adjacent to the descending aorta, with a second lesion in the left paracardiac region. A magnetic resonance (MR) study followed, which returned with a probable diagnosis of schwannoma (Figure 2 ). The larger left paraspinal mass was a 4-cm mass and appeared to arise from an exiting nerve root. The second was a $3-\mathrm{cm}$ mass with the same signal characteristics as the first mass.

A thoracotomy was performed to rule out and resect a possible posterior mediastinal tumor. At exploration, at least 6 scattered foci of beefy red appearance and well-formed individual sites of tissue were present within the left pleural cavity. The frozen section was consistent with splenosis. The majority of the tissue was left in situ to preserve any remnant of splenic function. A final diagnosis of splenosis was confirmed on the permanent pathologic specimens.

\section{Discussion}

Autotransplantation of splenic tissue in thoracic splenosis usually occurs as a result of trauma to the upper abdominal area with concomitant splenic damage and diaphragmatic tear. The newly implanted splenic tissue derives its blood supply from adjacent tissue and matures, generally in a benign fashion. There is evidence to suggest that in splenectomized patients this ectopic tissue might be beneficial. ${ }^{2,3}$

In our patient, radiographic, CT, and MR imaging led to the preliminary diagnosis of schwannoma primarily on the basis of location and appearance on chest MR imaging. Diagnosis of tho-

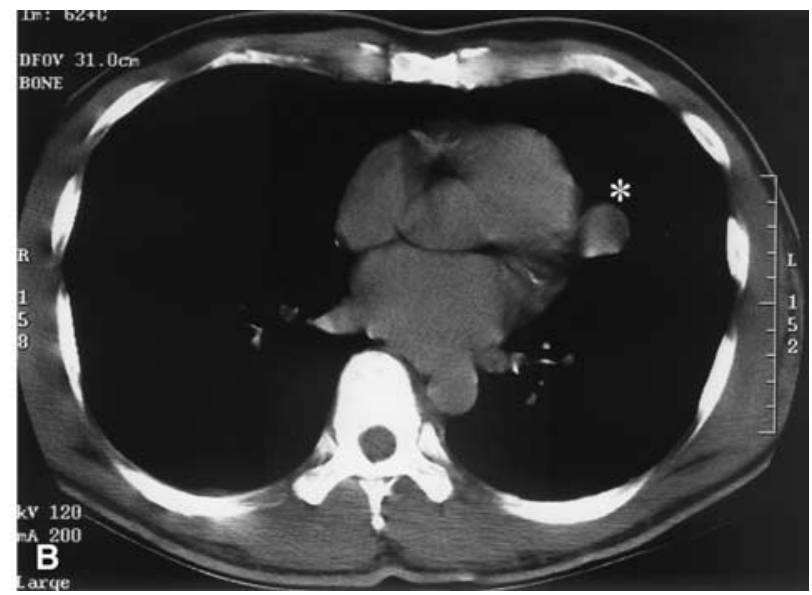

Figure 1. Preoperative CT images of the chest revealing masses consistent with a diagnosis of schwannoma (asterisk indicates mass). 

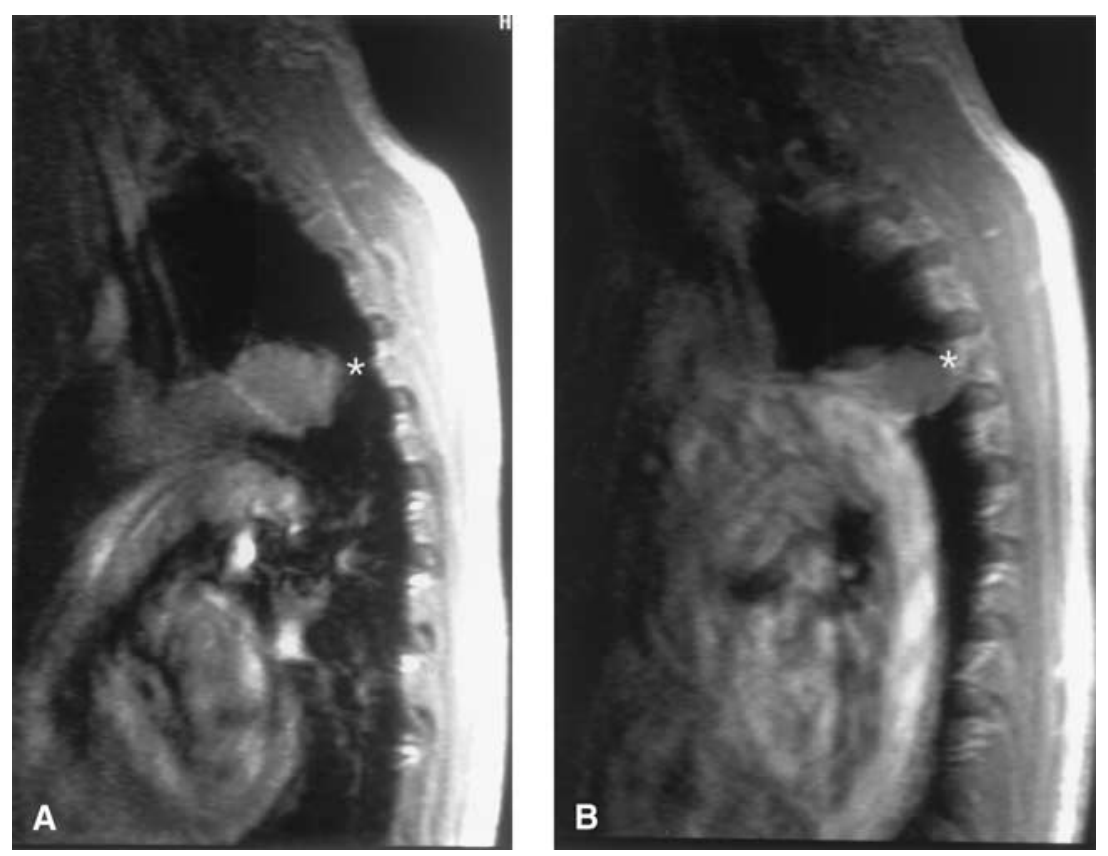

Figure 2. Preoperative MR images of the chest revealing masses consistent with a diagnosis of schwannoma (asterisk indicates mass).

racic splenosis was not made until the masses were visualized intraoperatively. When suspected, a preoperative diagnosis of thoracic splenosis can be confirmed by means of ${ }^{99 \mathrm{~m}} \mathrm{Tc}$ sulfur colloid scintigraphy, ${ }^{111}$ In-labeled platelet scan, or ${ }^{99 \mathrm{~m}}$ Tc-labeled heatdamaged erythrocyte study. ${ }^{4}$ It is thought that the latter 2 of the tests are the most sensitive and specific for the diagnosis of this interesting condition. ${ }^{5}$ Preoperative diagnosis of this phenomenon, in a patient after left upper quadrant trauma, is important because this is a benign condition with potential physiologic benefits. An accurate preoperative diagnosis, when possible, might avoid an otherwise unnecessary thoracic exploration.

\section{References}

1. Miller JS, Nunez A, Espada R. Thoracic splenosis 25 years after gunshot wound to the abdomen. J Trauma. 2001;50:149-50.

2. Schwartz AD, Goldthorn JF. "Born-again spleens" and resistance to infection. N Engl J Med. 1978;299:832-3.

3. Pearson HA, Johnston D, Smith KA, Touloukian RJ. The born-again spleen. Return of splenic function after splenectomy for trauma. N Engl J Med. 1978;298:1389-92.

4. Naylor MF, Karstaedt N, Finck SJ, Burnett OL. Noninvasive methods of diagnosing thoracic splenosis. Ann Thorac Surg. 1999;68:243-4.

5. Buchino JJ. Thoracic splenosis. South Med J. 1998;91:1054-6. 